\title{
Strategies for reducing expenditures in Iran's health transformation plan: A qualitative study
}

\author{
Keivan Rahmany ${ }^{1}$, Maryam Barati ${ }^{2}$, Masoud Ferdosi ${ }^{1}$, Amir Rakhshan ${ }^{3}$, Ali Nemati*4 \\ Received: 11 Jun 2018 \\ Published: 20 Oct 2018
}

\begin{abstract}
Background: Iranian health system underwent a series of reforms entitled Health Transformation Plan (HTP) in 2014. The plan started with packages that have imposed financial burden and increased expenditure in the health system. This study aimed to identify strategies and solutions to reduce expenditures in HTP in Iran.

Methods: To conduct this qualitative study, the researchers held 15 semi-structured interviews with prominent experts in the research arena in 2018. Content analysis was used to analyze the data using MAXQDA 10 software.

Results: Data collection yielded 9 main topics, including purchase and provision medicine, prescription, purchase and use of equipment, diagnostic medical services, referral system, human resources, physical space, payment system, and modifying and increasing base salaries.

Conclusion: In Iran's health system, some aspects of HTP wasted resources, eg, the waste of resources in the service delivery system; thus, policymakers should consider proper strategies to control the costs based on the nature of their implementation.
\end{abstract}

Keywords: Cost control, Health transformation plan, Qualitative study, Iran

Conflicts of Interest: None declared

Funding: Health Management and Economics Research Center, Iran University of Medical Sciences, Tehran, Iran

\section{*This work has been published under CC BY-NC-SA 1.0 license.}

Copyright $($ Iran University of Medical Sciences

Cite this article as: Rahmany K, Barati M, Ferdosi M, Rakhshan A, Nemati A. Strategies for reducing expenditures in Iran's health transformation plan: A qualitative study. Med J Islam Repub Iran. 2018 (20 Oct);32:102. https://doi.org/10.14196/mjiri.32.102

\section{Introduction}

The evolving needs of the societies, which should be met by the health system, have forced the systems to change throughout the world (1). However, the worldwide increase in the cost of the health systems has turned into one of the main concerns of managers and decision-makers of the health systems around the world (2). High expenses of health services, in many cases, have caused financial pressure for households and pushed them towards poverty and economic collapse (3). Thus, increasing the effectiveness of health services, justice, sustainable financing, and management improvement is the goal of health system reforms (4). The Iranian health system, like

\section{Corresponding author: Ali Nemati, ali_nemati96@yahoo.com}

1. Health Management and Economics Research center (HMERC), Isfahan University of Medical Sciences, Isfahan, Iran

2. Student Research Committee, School of Management and Medical Informatics, Isfahan University of Medical Sciences, Isfahan, Iran

3. Department of Foreign Languages, Tehran University of Medical Sciences, Tehran, Iran

4. Health Management and Economics Research Center, Iran University of Medical Sciences, Tehran, Iran many others, has encountered increasing costs. Health care expenditure has increased from $2.77 \%$ GDP in 2006 to $6.78 \%$ in 2011 . In addition, out-of-pocket expenditures have increased from 50.3 in 2006 to 58.46 in 2011 (5). The Ministry of Health and Medical Education (MoHME), as the health system stewardship, has implemented HTP, which includes increasing health insurance coverage, increasing the quality of care, reducing out-of-pocket expenditures, improving primary health care, launching updated relative value units (RVUs) of clinical services, and updating tariffs to more realistic values. HTP aimed to achieve 3 goals: (1) provide financial protection, (2) create

$\uparrow$ What is "already known" in this topic:

About 10\% of GDP expenditures is spent in the health system in developed countries. Cost control has always been an important yet challenging issue in the health system. Health Transformation Plan (HTP) has caused financial burden and increased expenditure in the health system in Iran.

$\rightarrow$ What this article adds:

This study comes up with cost control strategies for 5 service packages of HTP in Iran. Managers, decision- and policymakers of Iran's health system may use these strategies to control HTP expenditures. 
equity in access to health services, and (3) improve service quality. The main objectives of the plan included increasing the health system responsiveness, reducing out-of-pocket payments, reducing the percentage of households with catastrophic costs, improving the outcomes of emergency services, and increasing natural childbirth (6-7).

Studies on HTP have mainly focused on the effects of HTP on the goals of the plan, including patients' financial participation (8-11). Also, it has been shown that despite concerns of sustainability and equity in financing the plan, it has caused high public satisfaction. Financing the plan, equity, and fair participation in the financing have been one of the major concerns of the policy-makers of this plan (12).

Although appropriate steps have been taken to reduce the payments of service recipients in HTP, no specific strategies have been employed to control expenditure and supply and demand. Hence, we have witnessed an increase in the direct or indirect waste of resources in the service provision system. The present study was conducted to investigate the direct increase in the expenditures of the Iranian health system in HTP.

\section{Methods}

In this qualitative study, the researchers interviewed 15 participants using semi-structured interviews. Purposeful sampling was used to select the participants who met the inclusion criteria.

Inclusion criteria for the present study included having 10 years of work and management experience and being involved in the implementation of HTP.

The main stakeholders in HTP were identified through document review and generally included the Health Insurance Organization, HTP policy informants in MoHME, executive authorities in hospitals, and the support staff of health at Iran, Isfahan, and Tehran Universities of Medical Sciences.

Table 1 demonstrate characteristics of the interviewers. In sampling, in accordance with the principles of qualitative research, saturation method was employed. Also, the quantified proportion of the executive and staff managers was considered. To put it differently, after 13 interviews, the researchers did not notice any new information yielded by the interviewees in the $14^{\text {th }}$ and $15^{\text {th }}$ interviews. Therefore, the study sample was restricted to 15 experts. The interviews were all recorded and transcribed by one of the researchers up to the data saturation point. Generally, interviews took 45 to 60 minutes. Interviewer and interview guideline training was done based on the research team's opinion.

A semi-structured interview was used for data collection and individuals were fully free to answer the interviewer. The interviews were performed based on the interview question guide that had previously been extracted from a pilot interview with one single informant and discussed in the research group. The interview consisted of questions about the content of HTP, possible deficiencies and cost wasting because of HTP, the cost wasting that was worsened by HTP, and complementary questions. The interview question form and content validity were upheld by 4 experts along with the piloting process. A person was oriented by the research team to do the interviews. MAXQDA 10 was used to analyze the content analysis of interviews, which included extraction and writing the interviews, data immersion, encoding, recording of reflective symptoms, and recording marginal symptoms and abbreviations. This was an inductive analysis. Data validity was assessed by an auditor who audited the compliance of research findings with the required criteria. This study was approved by Health Management and Economics Research Center, Iran University of Medical Sciences, Tehran, Iran (No. 28649), and necessitated addressing ethical concerns, including obtaining interviewees' prior informed consent, fully informing the interviewees about the goal of the research, and keeping the comments of the interviewees confidential.

\section{Results}

The sample consisted of 6 hospital managers, 3 insurance experts, 2 university staff, and 4 policy informants in MoHME. In this study, the first and third phases (13) of the HTP, including the patients' cost reduction in public hospitals, were analyzed. The results of this qualitative data analysis are presented in Table 2.

\section{Package of reducing payments for inpatients}

\section{Lack of proper supply chain for the provision of drugs} in the hospital: Because medicine is a sensitive commodity to the treatment of patients, managing the medicine circulation process has an essential role in health promotion. Some participants stated, "Before HTP, the patient was providing medical supplies from outside sources. HTP focused on this issue and imposed the responsibility on hospitals, but the procedures were not homogeneous among hospitals and it worked differently in different places." (P2).

Another participant declared, "The procurement of medicine from pharmaceutical companies is by no means used for bidding or contracting. Using these methods properly and having good interaction with hospital pharmacists during the purchasing process will significantly reduce the cost of medicine and will result in more appropriate medicines. "(P9).

\begin{tabular}{|c|c|c|c|c|c|}
\hline \multicolumn{2}{|c|}{ Characteristic } & \multirow{2}{*}{$\frac{\mathrm{N}}{13}$} & \multicolumn{2}{|l|}{ Characteristic } & \multirow{2}{*}{$\frac{\mathrm{N}}{4}$} \\
\hline Sex & Male & & Age & $30-40$ & \\
\hline & Female & 2 & & $40-50$ & 7 \\
\hline \multirow{4}{*}{ Education } & Master of sciences & 1 & & $50-60$ & 4 \\
\hline & Medical Doctor & 3 & Work experience & $10-15$ & 2 \\
\hline & MD-MPH & 4 & & $15-20$ & 8 \\
\hline & $\mathrm{PhD}$ & 7 & & $20-25$ & 4 \\
\hline
\end{tabular}


Table 2. Main topics and identified wastes in HTP

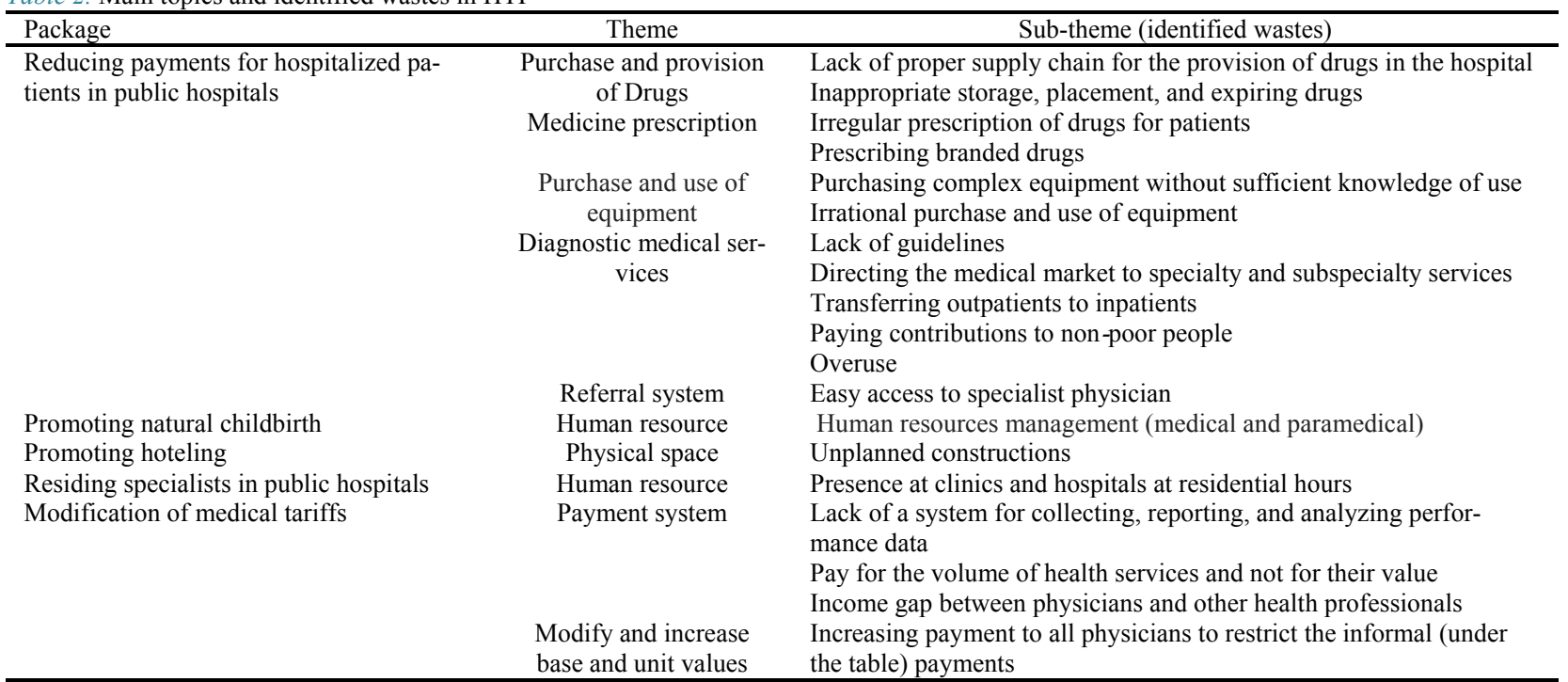

Inappropriate storage, placement, and expiring drugs: Because the cost of medicine is high, medicine storage is highly important, and a poor storage system will be very costly for the hospital. Some experts have pointed out that the wasting of medicines due to inappropriate placement and passing expiration date was one of the problems that became more intense after implementing HTP. "The small storage space led to problems with storage, inspection, poor preservation of medicines, and unconscionable medicine classifications." (P4). "Pharmacists are not well aware of determining cost-effective medicine delivery point and pharmacy management system, which in turn leads to shortage of medicines and passing expiration dates." (P12).

Irregular prescription of drugs for patients: Irregular prescription of medicines, especially antibiotics, and their overuse increase the bacterial resistance and impose irreparable financial and clinical costs on the health system. Many interviewees acknowledged that with the commencement of HTP, irregular prescription of hospital medicines became more common. "Physicians engage in irregular prescription of medicine in all situations knowing that it is supported by health subsidies and provides cash flow." (P7).

Prescribing branded drugs: Medicine quality is an entirely technical and scientific issue. Permission to produce a medicine is given when it has the same quality of the original brand, and this is considered as a quality point, then, the relevant documents are collected and controlled. "There is a biased viewpoint about the quality of medicines manufactured in Iran versus those manufactured in other countries. Many people think that foreign-branded medicines have a higher quality and efficacy compared to domestic-branded medicines, and prescribing foreignbranded medicines by physicians also confirms this false belief. "(P6).

Purchasing complex equipment without sufficient knowledge of use: Medical equipment is one of the 3 main pillars of the hospital and is one of the main bottlenecks in hospital costs. The most important factor contributing to the loss of hospital resources is purchasing complex equipment without the necessary expertise. Some participants have pointed out that complex technologies have been unused and unnecessary. "We spent a lot on updating hospital equipment. However, much of the equipment was damaged quickly because we did not have a good maintenance system, and it takes a long time to re-enter them to the cycle." (P8).

Irrational purchasing and use of equipment: The process of purchasing medical equipment starts from needs assessment and includes the purchase and installation of the equipment. "It was important to use a specific framework in all hospitals at the stage of analyzing and purchasing equipment; however, budgeting was not done effectively and sufficiently." (P1).

Lack of guidelines: When there is no guideline, providers can make irrational decisions, which can lead to discrepancy in outcomes and costs. Many participants pointed out that lack of guidelines has been a major challenge in implementing HTP and has led to an unnecessary increase in costs. "Differences in statistics and number of insured persons, and lack of clinical guidelines have made HTP problems more complicated." (P8).

Directing the medical market to specialty and subspecialty services: In all policies, priority is always given to preventive services; however, HTP has exacerbated this trend. "One reason for induced demand is the excessive use of specialty and subspecialty services that is flowing in the context of HTP and will impose great costs on the health system." (P4).

Transferring outpatients to inpatients: In a good health system, the care depends more on self-care because this pattern (move to self-care services) leads to reducing health expenditures. Some interviewees stated that HTP has raised the incentives for providers to provide inpatient services. "One of the weaknesses of HTP that has been observed is that to reduce the cost of diagnostic services, doctors recommend patients to be hospitalized, so the burden of outpatient care is transferred to the hospital." (p3, p13). 
Paying contributions to non-poor people: One of the goals of HTP was to achieve universal health coverage. "In the plan, the number of uninsured people was estimated to be between 5 and 6 million. Then, the strategy for insuring these people was to identify and insure them free of charge. As a result, 10 million people were insured and more than 3 million were in the waiting list to receive health insurance." (P 9).

Over Use (high level of demand from the customer and induced demand): This issue was raised by most interviewees as the main challenge of HTP. "The current policies have led to the emergence of induced demand and increased consumption, induced demands, and also increased the ordering of tests and other clinical and paraclinical processes without scientific justification." (P15). "My main concern about HTP is that it has increased consumption rate both in the field of equipment and medicine." (P13).

Easy access to specialist physicians: HTP has led to the provision of specialty and subspecialty services. In the first phase of the plan, along with reducing payment package, the visit quality improvement package was implemented. This package aimed to reduce the share of payment paid by the patients and to improve the quality of the visits when patients referred to specialist physicians. "Equipping public hospital clinics with specialist physicians facilitated access to specialist physicians and reduced patients' willingness to refer to general physicians.

\section{Promoting natural childbirth}

Human resources management (medical and paramedical): Unequal distribution of human resources in the health systems is a global phenomenon and occurs in a variety of dimensions, including inappropriate distribution of skills, specialty and subspecialty, and inappropriate institutional and gender distribution (14). Experts believed that one of the problems that led to lower cost efficiency in this package is inappropriate distribution of human resources across the country. "The shortage of medical and paramedical staff in different villages and cities of the country on the one hand and the large number of graduates of these fields in big cities and provincial centers on the other have caused disturbance in the provision of services to residents of deprived areas." (P7).

\section{Promoting hoteling}

Unplanned construction: The hospitals' infrastructure in the country is very old. In the unofficial reports of the Ministry of Health, nearly $60 \%$ of hospital beds need to be replaced. At the beginning of HTP, these packages were financed well and one of the concrete achievements was reconstruction of projects. However, most of these reconstructions took place outside of their principled process and brought many financial commitments to the plan. "The regular development program of the hospitals was interrupted by this plan. It can be said that all contributing organizations had their role in these problems, and thus reassessment of the plan is necessary." (P3, P11).

Residing specialists in public hospitals
Presence at clinics at residential hours: Based on the requirements in the residency program, emergency medicine specialists, internists, and surgeons will be present in hospitals, according to the instructions and with the written permission of the academic staff of HTP. The resident physician is responsible for making decisions about inpatients and emergency patients, performing all required diagnostic treatments, and managing hospital beds. The problems in this area were mentioned by one of the participants, "Lack of human resources and physician shortage have forced physicians to reside in clinics in the designated hours." (P5).

\section{Modification of medical tariffs}

Because of low tariffs and small contribution of the physicians from their earned income, informal payments, insurance companies' low coverage and support, and transfer of patients from the public sector to the private sector were prevalent prior to the implementation of HTP. However, illegal payment decreased through implementing the health transformation plan and increasing doctors' contribution (14). The hospitals' earnings doubled due to modification of medical tariffs.

Lack of a system for reporting, collecting, and analyzing performance data: To implement a performance-based payment system, we need a precise and fair performance evaluation framework. Many experts acknowledged that performance-based payment has been faced with many challenges and that their computed infrastructure was not accurate because our evaluation systems do not function properly. "The GHASEDAK Plan was inefficient and nothing was practically added to the amount of nurses' fee-for-service with the implementation of this plan; and in the new system, this rate increased from $27.5 \%$ to $29.5 \%$ of specialist performance payment." (P12). "This plan will deprive employees of their care responsibilities and it will keep them busy collecting points for themselves and their respective departments and hospitals." (P 15).

Pay for the volume of health services and not for their value: Different payment systems have their own advantages and disadvantages. Many participants pointed to an increase in resource waste in the present system after the implementation of HTP. "You can't talk about the performance as long as you pay for the services. The system, itself, provides incentives for corruption and gaining greater profit. Nowadays, more resources have entered the health system and this has caused a greater motivation for profiteering and making more financial benefit." (P1)

Income gap between physicians and other health professionals: Motivational theories emphasize the importance of employees' sense of justice to the fairness of the organization's behavior. Also, it is argued that if employees feel that they are treated unfairly, they would try to establish justice. Performance-based payment system and tariff modification have increased doctors' income considerably compared to nurses and other hospital medical staff. "Unfair income distribution in performance-based payment has led to increased wage disparities between 
doctors and nurses and even among nurses." (p11). "Implementation of this plan and its different computational formulas for physicians, with a minimum wage of 30 million Rails for specialists and 15 million Rials for general physicians, caused inequality and discrimination in medical staff payments and challenged the overall policy of the health system." (P9).

Increasing payment to all physicians to limit informal payments (under the table): Informal payment issues exist in all health systems and increase the amount of out-ofpocket payments. In HTP, the decision was made to raise payments to physicians to reduce their willingness to request informal payments. "Not all physicians got informal payments; eventually, $10 \%$ of them received these informal payments and it was not a big deal, but HTP increased the payment to all doctors, which wasted a large portion of HTP resources while it was not needed." (P4).

\section{Discussion}

Increasing health care expenditures over the last few decades has been one of the main concerns of health care policy-makers. Managers need to understand cost control strategies to improve their management and leadership, which include identifying and analyzing the circumstances and adjust leadership style based on each unique situation (14-15).

The immediate problem at the beginning of HTP was the large amount of out-of-pocket payment by patients. Financial protection is one of the basic goals of the health system (11). Irrational problem solving and injections of money into the health system imposed more cost on the health system. As demonstrated in the findings, many of the losses occurred in the first package of HTP.

Different studies have sought to organize the optimal health care services theoretically and experimentally, although they are mostly theoretical. Some studies have focused on service delivery and have responded to the question that how the organization and optimal financing of services are designed to balance quality and cost (16). HTP has caused new costs and changed payment shares in line with main objectives. In general, the services provided by HTP are as follow: (1) covering some services or medicines not previously covered by insurance, (2) transferring a high percentage of inpatient payments in public hospitals affiliated to MoHME to the health system (in the form of health subsidies), (3) improving hospital information management system and medical records, (4) promoting natural childbirth, (5) promoting equipment and services in public hospitals, (6) residing specialist physicians in hospitals, (7) improving quality of visits in public hospital clinics, and (7) changing medical tariffs and the payment system of doctors and hospital staff. All the above-mentioned changes imposed costs on MoHME and were financed from targeted subsidies and the added value of goods and services. Moradi Lakeh stated that HTP had a positive social reaction and that the overall satisfaction of the health transformation plan had been high, although there are concerns about the sustainability and equity of financing this plan. Past experience with the development of insurance coverage has shown that it could lead to recurrent financing and may be a threat to justice (12).

The wasted items in the field of medicine included the lack of proper supply chain and circulation of medicine in hospitals, large number of prescription medicines, irrational prescription of medicines, prescription of foreign medicines similar to domestic ones, and improper storage of medicines. Based on a study conducted in Japan, which has the highest medicine use per capita in OECD countries, the use of generic medicines has been considered as a method of reducing medicine costs (17).

In the field of equipment, the most significant losses were the purchase of complex equipment without required expertise and irrational purchase and use of equipment. Supply chain management in hospitals involves an internal chain (e.g., patient care unit, hospital ward, patient, etc.) and an external chain (e.g., salespersons, constructors, distributors, etc.). The supply chain involves purchasing, distributing, and managing suppliers, which includes a continuous flow of materials and services for the health supply chain (18). In developing countries, use of medical equipment is unfavorable because of mismanagement, which includes ineffective mechanisms, and bad purchase terms, maintenance, training, and technology utilization.

Due to bad purchase terms of medical equipment, loss of resources in this area continues and remains to be solved (19). Insufficient knowledge about the proper application of technology is one of the main reasons for low productivity in Iranian hospitals (20). Resource limitation in the health sector is being further devoured by the expensive technologies and high maintenance expenditures. Therefore, decisions and policies about adopting new technologies should be made based on national needs and demands, available resources, and the best evidence (21-22).

The major waste of resources in diagnostic and medical services caused by HTP were lack of guidelines, the shift to specialized services, providing health coverage for nonpoor people, and unnecessary diagnostic treatments in hospitals. When a plan is designed to reduce the cost of customers in any business, it will make people use this opportunity even in most cases without real needs. HTP has led to a general increase in patients' visits to public hospitals (23). In Haghshenas study, the following challenges were raised after implementation of HTP: an increase in the induced demand of physicians for paraclinical services, medicines, and equipment, and an increase in the number of inpatients because of reducing hospital costs (24). Faridfar et al found that the rate of admission in hospitals and in paraclinical sections has increased after the implementation of HTP (25).

The identified waste in the referral system included easy access to specialized physicians and patients' reluctance to refer to general physicians. The family physician-based approach, referral system, and primary care have been similar approaches in different health systems to control the cost of specialty services over the past years (26).

The waste arising from or aggravated by HTP to promote natural childbirth included inappropriate distribution of medical and paramedical personnel. According to different studies, $85 \%$ to $90 \%$ of births can be done naturally. The 
goal of the World Health Organization (WHO) is to achieve a cesarean delivery rate of $15 \%$; however, this rate is close to $50 \%$ in most developing countries (27). WHO has announced that there are no justifications for a cesarean delivery rate above $15 \%$ (28). Undoubtedly, one of the most important issues in the field of making meaningful reforms in the health system is the attention to the issue of human resources. The findings of Shaham et al showed that most of the studied hospitals before and after HTP had a lack of paramedical staff and that the effects of HTP on the lack of paramedical staff in hospitals were different (29). The results of the present study implicitly refer to abovementioned cases. Furthermore, given the lack of necessary infrastructure to facilitate or promote natural child birth, the implementation of natural childbirth package in HTP did not help to promote natural childbirth in the country.

In the package of residing specialists in public hospitals, one of the problems was insufficient time during which the residents attended the hospital. During residency, the presence of a specialist physician in public hospitals $24 / 7$ provides emergency patients with the opportunity to have access to specialist physicians (30). The results of Mousavi Rigi study indicated that after the execution of residency program of HTP, there was an improvement in the time needed for making decisions about a patient's course of treatment and it happened within 6 hours, and the discharge rate of the patients also improved (31).

According to the results of previous studies, this loss is debatable in 2 ways: first, the absence of a doctor reduces the effectiveness of the program and does not achieve its goals; second, more costs are incurred without any significant changes to the health system.

With respect to the modification of medical tariffs, the followings were the results of HTTP: (1) disruptions in the income levels of specialists; (2) lack of monitoring mechanisms; (3) payment for services volume; (4) imbalance payments to physicians and other medical staff; and (5) increase in the payments of all physicians. Considering that human resources play a central role in hospitals, these employees work according to the state laws and regulations, and thus the inadequacy of wages or unfairness of the payment system lead to their dissatisfaction, absenteeism, detention, strike, and grievance (32). Therefore, a fair payment system is one of the most important factors in promoting the productivity of the organization and its human resources (33). Tavakoli et al study showed that all organizations and individuals who mobilize health care credits should decide on what institution or individuals to pay, why should these resources be paid, what payment should be made and in what ways? and how should the monitoring be conducted to have the least violations? (34).

Informal payments have reduced health equity and motivation for service providers to have better quality, and they also led to corruption in the health system; as a result, incorrect decisions and improper policies which have been made based on this information became disruptive factors for reform in the health system (35). Informal payments are considered as the main source of health care financing and a serious obstacle for health care reforms in many developing countries (36). Payments to all physicians were increased by modification of the medical tariffs to deal with informal payments. However, only a small proportion of physicians used to receive informal payment before HTP, and increasing payment to all doctors, increased the costs and reduced the efficiency of the health system. This study showed that the ratio of informal payments to physicians declined after the implementation of HTP (37).

\section{Conclusion}

HTP and any reforms in the health system will be accompanied by new decisions regarding the costs, and thus the resources loss should be identified and controlled according to the nature of their implementation. The present study found that HTP has caused or intensified losses in financial and human resources in the health system of Iran in the following aspects: (1) medicine and equipment, (2) the use of health and diagnostic services, (3) referral system, (4) human resources for promotion of natural childbirth, (5) residing of specialists, and (6) reimbursement to physicians.

\section{Acknowledgments}

This study was supported by Health Management and Economics Research Center, Iran University of Medical Sciences (IUMS), Tehran. Iran (No.28649). The authors would like to thank all participants for their kind contributions to this project.

\section{Conflict of interests}

It is hereby declared by the authors that there exists no conflict of interests with other parties in the present study.

\section{References}

1. Shadpour K. Health sector reform in Islamic Republic of Iran. J Qazvin Univ Med Sci. 2006;10(3):7-20. (Persian)

2. Davari M. Economic challenges of Iran health system. Health INF Manag. 2011;8(7):915-17. (Persian)

3. Mehrara M, Fazaeli A, Fazaeli A. Health finance equity in Iran: An analysis of household survey data (1382-1386). Journal of Health Administration. 2010;13 (40):51-62. (Persian)

4. Mastaneh Z, Mouseli L. Health Information Position in New Reform of American Health Care System. Health Inform Manag. 2011;8(1): 111. (Persian)

5. Mehrolhassani M, Najafi B, Yazdi Feyzabadi V, Haghdoost A, Abolhallaje M, Ansari M, et al. Total Health Expenditures and Proportion of Out-Of-Pocket Payments in Iranian Provinces; 20082014. Iranian Journal of Epidemiology. 2017;12:1-12 (Persian)

6. Haghdoost AA, Mehrolhassani MH, Khajehkazemi R. Monitoringindicators of Iranian health system reform plan. Hakim Res J. 2013;16(3):171-81. (Persian)

7. Akhondzade R. Health system transformation project, an opportunity or a threat for doctors (editorial). Anesthesiol Pain. 2014;5(1):1-2.

8. Rezaei S, Moradi K, Akhgar A, Bazyar M, Heidari P. Hospitalcost associated with pediatrics urinary tract infection:Before and after health sector evolution program in the Westof Iran. Int J Pediatr. 2015;3(4-1):733-8.

9. Piroozi B, Mohamadi Bolban Abad A, Moradi G. Assessinghealth system responsiveness after the implementation ofhealth system reform: A case study of sanandaj, 2014-2015.Iran J Epidemiol. 2016;11(4):1-9.

10. Rezaei S, Rahimi foroushani A, Arab M, Jaafaripooyan E. Effects of the new health reform plan on the performance indicatorsof hamedan university hospitals. Sci J School Public Health. 2016;14(2):51-60.

11. Ghanbari A, Moaddab F, Heydarzade A, Jafaraghaee F, Barari F. 
Health System Evolution Plan; a New Approach to Health Care Delivery: The Challenge Ahead. Hakim Health Sys Res. 2017;20(1):18. (Persian)

12. Moradi-Lakeh M, Vosoogh-Moghaddam A. Health sector evolution plan in Iran; equity and sustainability concerns. Int J Health Policy Manag. 2015 Oct;4(10):637.

13. Emami Razavi SH. Health system reform plan in Iran: Approaching Universal Health Coverage. Hakim Health Sys Res. 2016;18(4):329335.

14. Boonla D, Treputtharat S. The Relationship between the Leadership Style and School Effectiveness in School under the office of Secondary Education Area 20, Social and Behavioral Sciences. 2014;112:991996

15. Azar FE, Asiabar AS. Does leadership effectiveness correlates with leadership styles in healthcare executives of Iran University of Medical Sciences. Med J Islam Repub Iran. 2015;29:166.

16. Ziebarth, Nicolas R., Assessing the Effectiveness of Health Care Cost Containment Measures (January 11, 2011). SOEPpaper No. 352. Available at SSRN: https://ssrn.com/abstract $=1750248$

17. Jones RS. OECD Economics Department Working Papers. OECD Publishing; 2009. Health-care reform in Japan: controlling costs, improving quality and ensuring equity. No. 739 .

18. Shih SC, Rivers PA, Hsu HS. Strategic information technology alliances for effective health-care supply chain management. Health Serv Manag Res. 2009;22(3):140-50.

19. Jadidi Ra, Bayati A, Arab MR. The effect of medical equipment maintenance management system implementation on Valie-Asr hospital costs situated in Arak: 2006. Arak Med Uni J. 2008;11(4):418.

20. Nabilou B, Yusefzadeh H, Rezapour A, Azar FE, Safi PS, Asiabar AS, et al. The productivity and its barriers in public hospitals: case study of ran. Med J Islam Repub Iran. 2016;30:316.

21. Sari AA, Ravaghi H, Mobinizadeh M, Sarvari S. The cost-utility analysis of PET-scan in diagnosis and treatment of non-small cell lung carcinoma in Iran. Iran J Radiol. 2013 Jun;10(2):61.

22. Sajjadi HS, Seyedin H, Aryankhesal A, Asiabar AS. A systematic review on the effectiveness of thermography in diagnosis of diseases. Int J ImagSyst Technol. 2013 Jun;23(2):188-93.

23. Majidi A, Mahmoodi S, Haji Adineh V. An Epidemiologic Study of Emergency Department Visits before and after Executing Health Sector Evolution Plan; a Brief Report. Iran J Emerg Med. 2017;4(3):134. (Persian)

24. Haghshenas M. Comparative study of goals, plans and challenges of health transformation plan in Iran, Turkey and United States of America. Assess Know Quart. 2017;9(31). (Persian)

25. Faridfar N, Alimohammadzade K, Seyedein S. Impact of healthcare overhaul plan on clinical, paraclinical, surgery indexes and on the patients satisfaction in Rasul Akram hospital in 2013-2014. Razi Med Sci J. 2015;22(140):92-9. (Persian)

26. Stone C, Rosella L, Goel V. Population health perspective on high users of health care: Role of family physicians. Canad Fam Physic. 2014;60(9):781-3.

27. Baghianimoghadam MH, Zolghadar R, Moghadam BB, Darayi M, Jozy $F$. Related factors to choose normal vaginal delivery by mothers based on Health Belief Model. J Educ Health Prom. 2012;1.

28. Amiri M, Raei M, Chaman R, Rezaee N. Investigating some of the factors influencing choice of delivery type in women working in Shahroud University of Medical Sciences. Razi J Med Sci. 2013;20(106):1-9.

29. Shaham G, Komeili A, Masoudi AI. Impact of healthcare overhaul plan on manpower distribution at selected hospitals affiliated to tehran university of medical sciences. J Healthcare Manag. 2016; 7(3):17-26

30. Aghajani M, Vaezi H, Shahrami A, Massoumi Gh, Rastegari M. The resident program of specialists in the hospitals of the Ministry of Health and Medical Education. Tehran .Ministry of Health and Medical Education Deputy of curative affairs. 2017:91. (Persian)

31. Mousavi-Rigi SA, Dorahaki M, Ebrahimi S. Comparison of Performance Indices of Emergency Departments before and after Implementation of Specialist Residency Program under the Health Sector Evolution Plan in the Hospitals of Bushehr University of Medical Sciences, Iran. Health Inform Manage. 2017;14(5): 205-10. (Persian)

32. Raeisi P, Alikhani M, Mobinizadeh M. Fee for Service (FFS) Payment on the basis of performance in Hasheminejad Hospital, Tehran. J Healthcare Manag. 2010;2(1):27-36.
33. Ardestani AS, Asiabar AS, Azar FE, Abtahi SA. The relationship between hospital managers' leadership style and effectiveness with passing managerial training courses. Med J Islam Repub Iran 2016;30:465.

34. Tavakkoli M, Karimi S, Javadi M, Jabbaria A. Reform Strategies to Improve Monitoring Performance and Infrastructure Implementation development of the New Scheme of Fee Guidelines in 2014 (A Qualitative Study). J Hospital. 2017;16(1):103-113. (Persian)

35. Khodamoradi A, Rashidian A, Aghlmand S, Arab M. Informal Payments in Health Sector and Policies to Deal with (A Review Study). J Hospital. 2015;14(3):23-32

36. Aboutorabi A, Ghiasipour M, Rezapour A, Pourreza A, Asiabar AS, Tanoomand A. Factors affecting the informal payments in public and teaching hospitals. Med J Islam Repub Iran. 2016;30:315.

37. Piroozi B, Rashidian A, Moradi G, Takian A, Ghasri H, Ghadimi T. Out-of-pocket and informal payment before and after the health transformation plan in Iran: evidence from hospitals located in Kurdistan, Iran. Int J Health Policy Manag. 2017 Oct;6(10):573. 